\title{
Layer-by-layer gelatin/chitosan polyelectrolyte coated nanoparticles on Ti implants for prevention of implant-associated infections
}

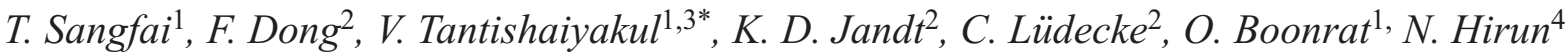 \\ ${ }^{1}$ Department of Pharmaceutical Chemistry, Faculty of Pharmaceutical Sciences, Prince of Songkla University, 90112 \\ Hat-Yai, Thailand \\ ${ }^{2}$ Otto Schott Institute of Materials Research, Friedrich-Schiller-University Jena, Löbdergraben 32, 07743 Jena, Germany \\ ${ }^{3}$ Nanotec-PSU Center of Excellence for Drug Delivery System, Faculty of Pharmaceutical Sciences, Prince of Songkla \\ University, 90112 Hat-Yai, Thailand \\ ${ }^{4}$ Theoretical and Computational Modeling Research Group and School of Pharmacy, Walailak University 80161, Nakhon \\ Si Thammarat, Thailand
}

Received 1 July 2016; accepted in revised form 14 September 2016

\begin{abstract}
Gelatin nanoparticles (Gb-NP) and layer-by-layer (LBL) coated NPs were developed to modify a Ti substrate surface to prevent implant-associated infections. Vancomycin (Van) was loaded into these materials to obtain GbV-NP and LBL-GbV-NP. The size of the GbV-NP $(277.4 \pm 1.4 \mathrm{~nm})$ was smaller than that of LBL-GbV-NP $(710.2 \pm 4.6 \mathrm{~nm})$ but both had a spherical shape. These coated materials showed no cytotoxicity and facilitated better cell proliferation by osteoblast-like cells compared to the bare Ti. This was probably due to the roughness of the coated NP that enhanced cell attachement to the surface. Both coated materials showed antibacterial activity against $S$. aureus. The release of Van from GbV-NP was higher from LBL-GbV-NP and this corresponded to their antibacterial activity. Furthermore the release profile of Van showed a sustained release. Thus both materials should be able to prolong the protection of implant-associated infections to the bone.
\end{abstract}

Keywords: nanomaterials, coatings, biocompatible polymers, vancomycin, Ti implantation

\section{Introduction}

Titanium (Ti) and Ti alloys have been extensively used in dental and orthopaedic areas as implants due to their biocompatibility, low density and good mechanical properties. However, unmodified Ti may host bacterial infections after implantation [1]. These infections often lead to implant failure, removal of implants, higher treatment costs, and also increased patient morbidity or in some cases perioperative mortality. Infections must be treated carefully with antimicrobial agents. High systemic doses of some agents may cause various side effects including nephrotoxicity and ototoxicity. Applications of local antimi- crobial agents have generated much interest as an alternative drug delivery system. Basically, local drug delivery will allow for a better control of drug application directly at the required site/location for an extended period of time.

Various antibiotic coatings on Ti have been investigated to provide a local antibacterial delivery to a $\mathrm{Ti}$ implant [2]. Most studies have involved incorporating antibiotics into numerous types of materials coatings such as carbonated hydroxyapatite [3] chitosan (Chi) [4] and tetraethylorthosilane [5] either directly, as a film or a layer-by-layer (LBL) coating [6-8]. Modifications of titanium implant surfaces by cova-

$\overline{{ }^{*} \text { Corresponding author, e-mail: vimon.t@psu.ac.th }}$ (C) BME-PT 
lently bonded antibiotics have also been employed $[2,9]$. Recently nanoparticles (NPs) have been applied as an alternative coating on Ti implant surfaces [10-12]. These included the use of chitosan nanoparticle-based coating [10], such as antibiotic-loaded silk protein fibroin NPs and antibiotic-loaded electrospun coatings [12]. Nanostructure modifications of the Ti surface have several advantages. The increase of the Ti surface roughness by NPs has provided better cell attachment and enhanced the in vitro bone bonding capacity $[11,13,14]$. In addition, it was shown that nanostructured surfaces did reduce inflammation compared to a flat surface [15]. Therefore surface modifications of Ti can improve cell attachment and probably osseointegration by connecting between the Ti surface roughness of the NP coating and the newly formed bone tissue.

Coating by LBL is an advanced technique to assemble polyelectrolyte multilayer (PEM) films onto polymer surfaces, mesoporous silica nanoparticles and Ti surfaces by sequential adsorption of oppositely charged polyanions and polycations via electrostatic interactions [16-22]. This technique is expected to provide a more durable coating due to the electrostatic attractions between layers and between the first layer and substrate [6]. Several methods have been employed for LBL coating including centrifugation, microfluidics, dipping, casting and spraying $[23,24]$. Gelatin is a hydrophilic, biodegradable protein derived from collagen by partially acidic (type A) or alkaline (type $\mathrm{B}, \mathrm{Gb}$ ) hydrolysis [25]. Chitosan is a polysaccharide comprised of glucosamine and $\mathrm{N}$ acetylglucosamine. Both gelatin and chitosan have been extensively investigated and used in pharmaceutical applications due to their biocompatibility and biodegradable properties. Chitosan also has a feeble antibacterial activity [26, 27]. Recently, chi/ gelatin as a polycation/polyanion for use as an LBL system has been successfully prepared [6]. This hydrophilic coating may provide an antiadhesive surface and reduce biofilm formation on the surface [28]. The electrostatic LBL self-assembly using Chi/ Gb was thus employed in this study.

The objective of this study was to develop an effective antibiotic, vancomycin $\mathrm{HCl}$ (Van), loaded Gb$\mathrm{NP}(\mathrm{GbV}-\mathrm{NP})$ to produce the surface roughness that was subsequently coated with polyelectrolytes (Chi/ $\mathrm{Gb})$ using LBL assembly to prevent bacterial infection. This PEM coating NP (LBL-GbV-NP) could probably provide a sustained release of the Van. All coating materials (Gb-NP, GbV-NP, LBL-GbV-NP) were characterized for their particle sizes, zeta potential and morphology. The interactions between Van and the NP were also investigated using attenuated total reflection Fourier transform infrared spectroscopy (ATR-FTIR). The amount of Van contained in the coating materials was analyzed. Cell proliferation and cytotoxicity were determined using osteoblast like cells (CAL-72). Antibacterial activity against $S$. aureus was also assessed. In addition the release of Van from the coating materials was examined.

\section{Experimental}

\subsection{Chemicals}

Gelatin type B (Gb, bovine bone type-B gelatin), Van, $25 \%$ glutaraldehyde, chitosan (medium molecular weight with a deactylation degree $>85 \%$ ) and poly (etheyleneimine) (PEI, $M_{\mathrm{n}}=65000$ ), were purchased from Sigma-Aldrich Chemical Co. St. Louis, Mo. Flat titanium thin films were deposited on borofloat glass substrates via electron-beam deposition. All films were deposited with a film thickness of $200 \mathrm{~nm}$.

\subsection{Preparation of nanoparticles}

The Gb-NP and GbV-NP were prepared using a desolvation technique as previously described $[29,30]$. A $20 \mathrm{~mL}$ aqueous solution of $\mathrm{Gb}(100 \mathrm{mg})$ with Van $(20 \mathrm{mg}$ ) and without Van were prepared and adjusted to $\mathrm{pH} 6-10$ by the addition of $\mathrm{NaOH}(0.1 \mathrm{M})$. Then the solution of $\mathrm{Gb}$ or $\mathrm{GbV}$ was stirred $(600 \mathrm{rpm})$ at $50^{\circ} \mathrm{C}$ until clear. To induce the desolvation process, acetone was added until a permanent faint turbidity was obtained. Subsequently, glutaraldehyde solution $(8 \% \mathrm{w} / \mathrm{v}, 250 \mu \mathrm{L})$ was added to harden the particles. The preparation was then stirred overnight at $1000 \mathrm{rpm}$. The obtained NPs (Gb-NP and GbV-NP) were separated from the solution by centrifugation at $9000 \mathrm{rpm}$ for $20 \mathrm{~min}$, then washed 2-3 times with distilled water, re-suspended and dispersed in $20 \mathrm{~mL}$ of distilled water for use. The optimal $\mathrm{pH}$ based on the zeta potential and size of the obtained GbV-NP

Table 1. Hydrodynamic diameters, polydispersity index and zeta-potential of GbV-NP prepared at various $\mathrm{pHs}$ $(n=3$, mean \pm SD $)$

\begin{tabular}{|r|c|c|c|}
\hline $\mathbf{p H}$ & $\begin{array}{c}\text { Diameter } \\
{[\mathbf{n m}]}\end{array}$ & $\begin{array}{c}\text { Polydispersity } \\
\text { index }\end{array}$ & $\begin{array}{c}\text { Zeta-potential } \\
{[\mathbf{m V}]}\end{array}$ \\
\hline 6 & $410.5 \pm 3.8$ & $0.222 \pm 0.015$ & $-19.7 \pm 0.4$ \\
\hline 8 & $277.6 \pm 1.2$ & $0.145 \pm 0.015$ & $-27.1 \pm 0.2$ \\
\hline 10 & $280.7 \pm 2.4$ & $0.177 \pm 0.015$ & $-26.7 \pm 0.3$ \\
\hline
\end{tabular}


was $\mathrm{pH} 8$ (Table 1) and this $\mathrm{pH}$ was employed for the NP preparation.

\subsection{Preparation of PEM shell surrounding nanoparticles}

The PEM shell coating on GbV-NP was carried out by coating the first layer with $\mathrm{Chi}$ and the second layer with $\mathrm{Gb}$. Chitosan (60 mg) was dissolved in $0.2 \mathrm{M}$ acetic acid $(30 \mathrm{~mL})$ to obtain the Chi concentration of $2 \mathrm{mg} / \mathrm{mL}$. The GbV-NPs $(100 \mathrm{mg})$ were then suspended in the Chi solution $(30 \mathrm{~mL})$ and stirred for $15 \mathrm{~min}$ at room temperature. Subsequently, the mixtures were centrifuged and washed with distilled water to obtain the GbV-NP/Chi coating. This procedure was also employed for coating the GbV-NP/ Chi with $\mathrm{Gb}$ by using the $\mathrm{Gb}$ solution $(2 \mathrm{mg} / \mathrm{mL})$ to obtain GbV-NP/Chi/Gb or LBL-GbV-NP.

\subsection{Preparation of nanoparticles coating on Ti surface}

The deposited Ti films were immersed in PEI solution $(5 \mathrm{mg} / \mathrm{mL})$ for $20 \mathrm{~min}$. This created a precursor layer with a stable positive charge to initiate the electrostatice force with Gb-NP, GbV-NP or LBL-GbVNP that had a negative charge. These NPs were deposited onto the Ti/PEI sheets by drop casting as previously described [10]. Briefly, $200 \mu \mathrm{L}$ of NP suspension was dropped onto the Ti/PEI and subsequently the coatings on the Ti/PEI sheets were ovendried at $37^{\circ} \mathrm{C}$ to obtain Ti/Gb-NP, Ti/GbV-NP and Ti/LBL-GbV-NP.

\subsection{Characterization of nanoparticles}

The suspension of NP $(100 \mu \mathrm{L})$ was dispersed in $2 \mathrm{~mL}$ of deionized water and hydrodynamic size measurements were performed using dynamic light scattering (DLS) on a Nano-ZS90 Zetasizer (Malvern Instruments Ltd. Co., UK). Zeta $(\zeta)$ potentials were measured also using a Nano-ZS90 Zetasizer in capillary cells. All the measurements were conducted at room temperature in triplicate.

The morphology of the oven-dried GbV-NP and LBL-GbV-NP on the Ti surface was characterized by SEM (S440i, Leica, Germany) at a magnification of $\sim 5000 \times$ operated at $20 \mathrm{kV}$. A Focused Ion Beam Scanning Electron Microscope (FIB-SEM, AURIGA 60 CrossBeam $^{\circledR}$, Carl-Zeiss AG, Oberkochen, Germany) was also employed for morphological characterization and cell proliferation on the samples.
The possible interactions between the Van and the utilized polymers were investigated using Fourier tansform infrared (FTIR) spectroscopy (FTIR spectrometer Alpha-P with evaluation software Opus, Bruker, Rheinstetten, Germany). Samples included Van and $\mathrm{Gb}$ powders and freeze dried samples of Gb-NP, GbV-NP and LBL-GbV-NP. The FTIR spectra were recorded (range: $375-4000 \mathrm{~cm}^{-1}$, resolution: $4 \mathrm{~cm}^{-1}$, number of scans: 24$)$ in an attenuated total reflection (ATR) mode.

\subsection{Determination of vancomycin entrapment in gelatin nanoparticles}

The entrapment efficiency (EE) of Van in GbV-NP was determined by the indirect method as previously described [10]. The GbV-NP were separated by centrifugation of the colloidal suspension for $20 \mathrm{~min}$ at $9000 \mathrm{rpm}$ at $25^{\circ} \mathrm{C}$ (section 2.2), and the free Van $\left(V a_{\text {free}}\right)$ in the supernatant was measured by UV-Vis spectrophotometry at $282 \mathrm{~nm}$ as previously described [31]. The \%EE was calculated according to Equation (1):

$\% \mathrm{EE}=\frac{\operatorname{Van}_{\text {tot }}-\operatorname{Van}_{\text {free }}}{\operatorname{Van}_{\text {tot }}} \cdot 100$

where $\operatorname{Van}_{\text {tot }}$ denoted the amounts of Van present in the solution.

\subsection{Vancomycin release study}

In vitro release studies of Van were carried out on the GbV-NP and LBL-GbV-NP coating on Ti/PEI. The coated materials were immersed in $1.5 \mathrm{~mL}$ of PBS (pH 7.4) in a 24 well plate at $37^{\circ} \mathrm{C}$ in the oven as previously described [11, 12]. At pre-determined time intervals, an aliquot $(500 \mu \mathrm{L})$ was withdrawn and the amount of Van was determined by UV spectroscopy at $282 \mathrm{~nm}$ as mentioned in section 2.6. A total volume of $1.5 \mathrm{~mL}$ was maintained by refilling with $500 \mu \mathrm{L}$ of the same medium after each withdrawal. All experiments were performed in triplicate.

\subsection{Antimicrobial test}

Antimicrobial activity of the coated samples was investigated based on an agar diffusion test (KirbyBauer antibiotic test [32] and Staphylococcus aureus MRSA 134/94 (methicillin-resistant strain) as a model organism.

S. aureus was pre-cultured for $12 \mathrm{~h}$ in LB (lysogeny broth) medium. Afterwards, the bacterial culture was 
diluted using physiological $\mathrm{NaCl}$ solution to obtain an optical density of 0.5 which according to the McFahrland standard roughly corresponds to $1.5 \cdot 10^{8} \mathrm{CFU}$ (colony forming units) per $\mathrm{mL}$. For preparation of the agar diffusion plate, $100 \mu \mathrm{L}$ of the bacterial suspension was added to $34 \mathrm{~mL}$ of molten medium-agar solution (at $56^{\circ} \mathrm{C}$ ) per plate. After cooling, the coated samples were placed upside down on the agar diffusion plates. The plates were incubated for $24 \mathrm{~h}$ at $37^{\circ} \mathrm{C}$. The experiments were performed in triplicate.

\subsection{Cell proliferation, cell viability test and scanning electron microscopy}

Cell proliferation on the coated samples was tested using osteoblast like cells (CAL-72, derived from human osteosarcoma ACC439; German Collection of Microorganisms and Cell Cultures DSMZ, Braunschweig, Germany). The cells were cultured in Dulbecco's modified eagles medium (DMEM, VWR International, Dresden Germany) containing 10\% fetal bovine serum, at $37^{\circ} \mathrm{C}$ in a humidified $5 \% \mathrm{CO}_{2}$ atmosphere. The coated materials ( $n=5$ replications) were placed in tissue culture poly(styrene) well plates and incubated with 10000 cells per $\mathrm{cm}^{2}$ (corresponding to 20500 cells per well) for 24 and $72 \mathrm{~h}$ (3 days) at $37^{\circ} \mathrm{C}$ in an humidified $5 \% \mathrm{CO}_{2}$ atmosphere. After incubation, the samples were gently washed twice with phosphate buffered saline (PBS) to remove nonadherent cells, trypsinated with $0.25 \%$ trypsin in PBS, and subsequently counted with a Z1 Coulter counter (Beckman Coulter GmbH, Krefeld, Germany). A plate well without sample was used as a control.

Cell viability was determined by the MTT assay. After culturing the cells for 3 days at $37^{\circ} \mathrm{C}, 5 \% \mathrm{CO}_{2}$, the medium was then removed; $200 \mu \mathrm{L}$ of methylthiazolyldiphenyl-tetrazolium bromide (MTT, Aldrich 135038, Sigma-Aldrich) solution $(5 \mathrm{mg} / \mathrm{mL})$ and $1.8 \mathrm{~mL}$ PBS were added to each well and incubated at $37^{\circ} \mathrm{C}$ for a further $4 \mathrm{~h}$. After discarding the MTT solution, the dark blue formazan crystals were dissolved by adding $100 \mu \mathrm{L}$ of dimethyl sulfoxide (DMSO) and quantified using a microplate reader (Biohit 830, Biohit ${ }^{\circledR}$, Helsinki, Finland) at $570 \mathrm{~nm}$. The results are reported as a percentage in comparison with a cell culture medium that was used as a control.

Osteoblast cells cultured on Ti/Gb-NP, Ti/GbV-NP and Ti/LBL-GbV-NP were evaluated by FIB-SEM after being cultured for 3 days. For the FIB-SEM im- aging, samples with adherent cells were gently washed twice with PBS to remove non-adherent cells and, afterward, fixed in glutaraldehyde solution $(2.5 \%)$ for $24 \mathrm{~h}$ at room temperature. After fixation, samples were washed for 30 min in PBS buffer solution and twice in distilled water. Subsequently, samples were dehydrated using an ascending ethanol series from 10 to $>99.9 \%$ with 30 min incubation per step and two times at $60 \mathrm{~min}$ for the last step. The dehydrated samples were dried by the critical point method (EMITECH K850, Quorum Technologies Ltd, East Grinstead, UK) and gold sputter coated (S150B, Edwards Ltd, Crawley, UK).

All data were expressed as mean values \pm standard deviation. Statistical analysis was performed using the IBM SPSS Statistics version 22 for Windows. Data were analyzed using one-way analysis of variance (ANOVA) followed by a Tukey post hoc test for multiple comparison tests.

\section{Results and discussion}

\subsection{Preparation and characterization}

As listed in Table 2, the hydrodynamic diameter of the Gb-NP was found to be $216.3 \mathrm{~nm}$ with a polydispersity index (PDI) of 0.052 and a zeta potential of $-29.9 \mathrm{mV}$ which were similar to previous reports [29, 33]. The PDI values were around 0.14 for the GbV$\mathrm{NP}, \mathrm{GbV}-\mathrm{NP} / \mathrm{Chi}$ and LBL-GbV-NP. In the same way as previous studies, the size and zeta potential values of the gelatin NPs were dependent on various parameters including temperature, $\mathrm{pH}$ and the concentration of glutaraldehyde [30, 33]. In this study, the glutaraldehyde concentration and temperature for preparing the NPs were the optimal concentration and temperature reported in the literature [30,33]. The size of the drug loading Gb-NP (GbV-NP) was slightly increased to $277.4 \mathrm{~nm}$ compared to the unloaded NP.

The Chi (polycation) coating onto the GbV-NP (negative charge) resulted in a larger size $(324 \mathrm{~nm})$ and the zeta potential was changed to a positive charge $(+27.2 \mathrm{mV})$. The GbV-NP/Chi was converted to a

Table 2. Hydrodynamic diameters, polydispersity index and zeta-potential of various nanopraticles prepared in three separate experiments (mean $\pm \mathrm{SD}$ )

\begin{tabular}{|l|c|c|c|}
\hline \multicolumn{1}{|c|}{ Samples } & $\begin{array}{c}\text { Diameter } \\
{[\mathbf{n m}]}\end{array}$ & $\begin{array}{c}\text { Polydispersity } \\
\text { index }\end{array}$ & $\begin{array}{c}\text { Zeta-potential } \\
{[\mathbf{m V}]}\end{array}$ \\
\hline $\mathrm{Gb}-\mathrm{NP}$ & $216.3 \pm 1.6$ & $0.052 \pm 0.033$ & $-29.9 \pm 0.6$ \\
\hline $\mathrm{GbV-NP}$ & $277.4 \pm 1.4$ & $0.144 \pm 0.018$ & $-27.0 \pm 0.3$ \\
\hline $\mathrm{GbV-NP/Chi}$ & $324.8 \pm 1.0$ & $0.144 \pm 0.017$ & $+27.2 \pm 0.8$ \\
\hline $\begin{array}{l}\mathrm{GbV}-\mathrm{NP} /(\mathrm{Chi} / \mathrm{Gb}) \\
\text { or LBL-GbV-NP }\end{array}$ & $710.2 \pm 4.6$ & $0.140 \pm 0.035$ & $-20.0 \pm 0.2$ \\
\hline
\end{tabular}




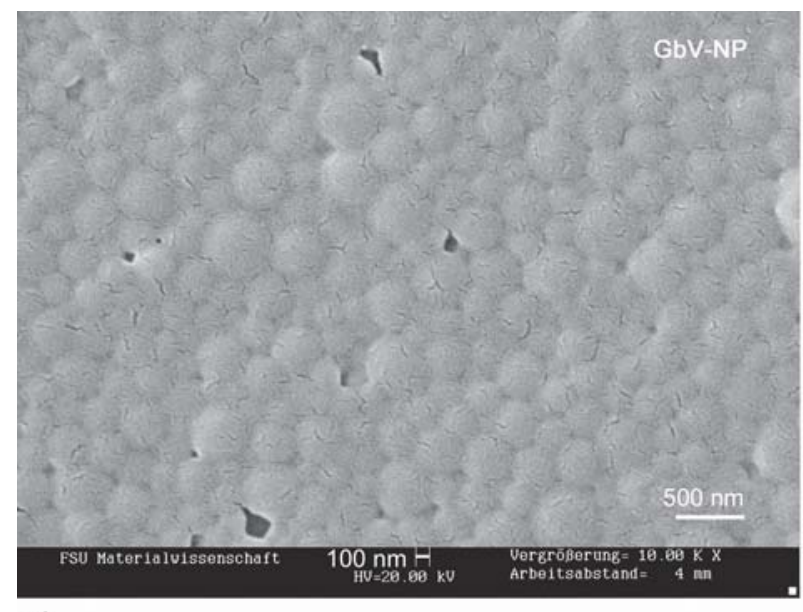

a)

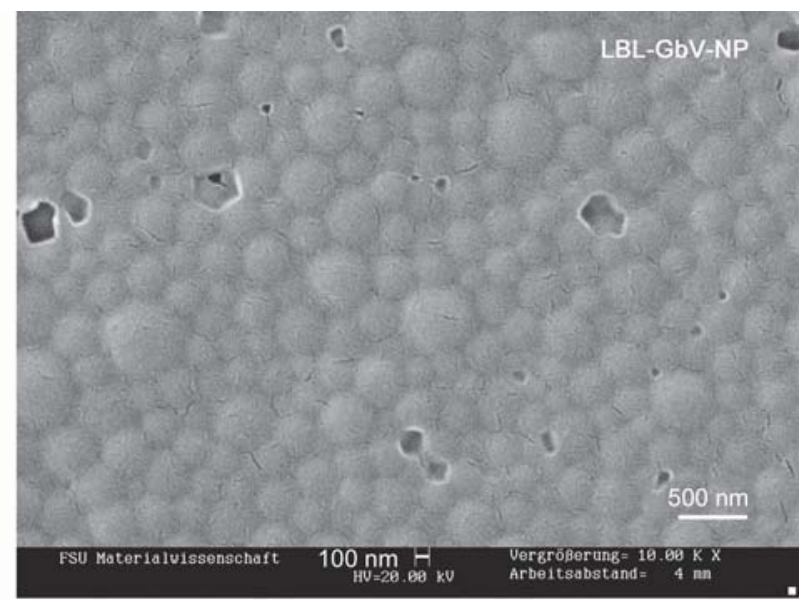

b)

Figure 1. SEM micrographs of GbV-NP (a) and LBL-GbV-NP (b)

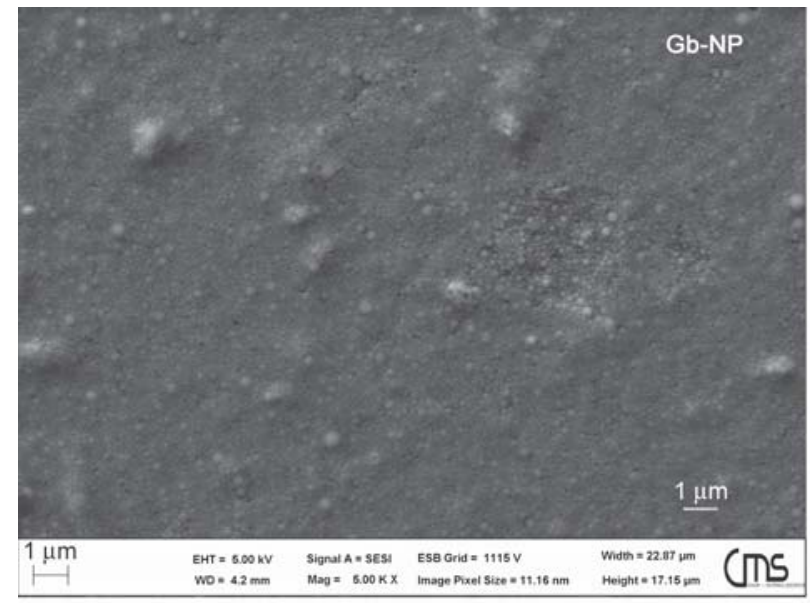

a)

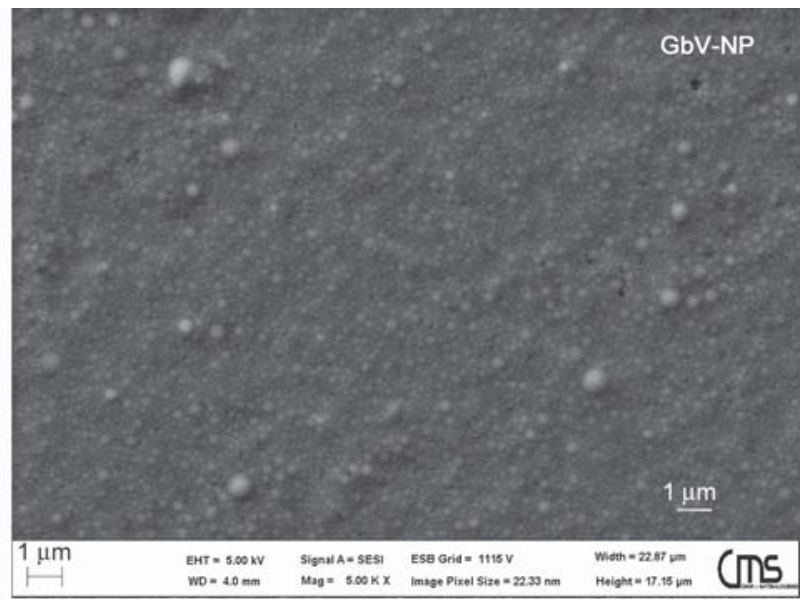

b)

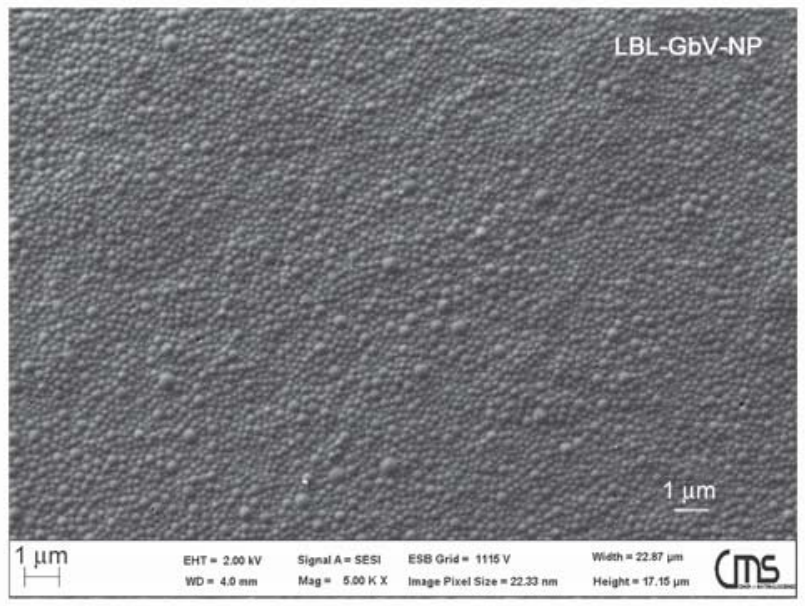

c)

Figure 2. FIB-SEM characterization of Gb-NP (a), GbV-NP (b) and LBL-GbV-NP (c)

negative charge $(-20 \mathrm{mV})$ again after being surrounded with $\mathrm{Gb}$ (polyanion). This confirmed that the assembly had taken place on the surface of the NPs. As shown in Figures 1 and 2, all the NPs (Gb-NP, GbV-NP and LBL-GbV-NP) had spherical shapes with a smooth surface. The Gb-NP, GbV-NP and
LBL-GbV-NP showed the same morphology but were of a slightly larger size after coating with the $\mathrm{Chi} / \mathrm{Gb}$ (LBL-GbV-NP). The Van and Chi/Gb coating did not cause any observed effect on the surface of the NPs. The NPs size ranges were in agreement with the mean particle size characterized by DLS. 


\subsection{ATR FTIR analysis}

Figure 3 shows the ATR-FTIR spectra of the Van, Gb, Gb-NP, GbV-NP and LBL-GbV-NP. The ATR-FTIR spectra of all samples showed broad $\mathrm{OH}$ bands of a phenolic or acid group from the Van and $\mathrm{Gb}$ at about $3200 \mathrm{~cm}^{-1}$. Due to their broad bands, the interactions at these functional groups of each sample could not be detected. The $\mathrm{C}=\mathrm{O}$ stretching bands of the Van and $\mathrm{Gb}$ were observed at 1647 and $1633 \mathrm{~cm}^{-1}$, respectively. The $\mathrm{C}=\mathrm{O}$ stretching of Gb-NP, GbV-NP and LBLGbV-NP appeared, respectively, at 1639, 1638 and $1637 \mathrm{~cm}^{-1}$, which were at a relatively lower wave number than Van. The spectra of all the NPs were comparable to that of $\mathrm{Gb}$ since $\mathrm{Gb}$ was the major component in the NPs. The slight difference of the NPs and Gb may be due to the cross-linked Gb caused by the glutaraldehyde in the NP preparation. The prominent bands of the Van powder were unfortunatly not detected in the spectra of the NPs containg Van, GbV-NP or LBL-GbV-NP. This may be due to the Van loading to only $11.6 \%$ in the NPs, based on the NP preparation and the \%EE of Van. Thus, the spectrum of the Van in the NPs was probably shielded by the $\mathrm{Gb}$ spectrum. Therefore, it may be difficult to conclude whether Van did interact with the Gb-NP. Nevertheless, the covalently attached Van to the Ti implant surfaces has previously been report-

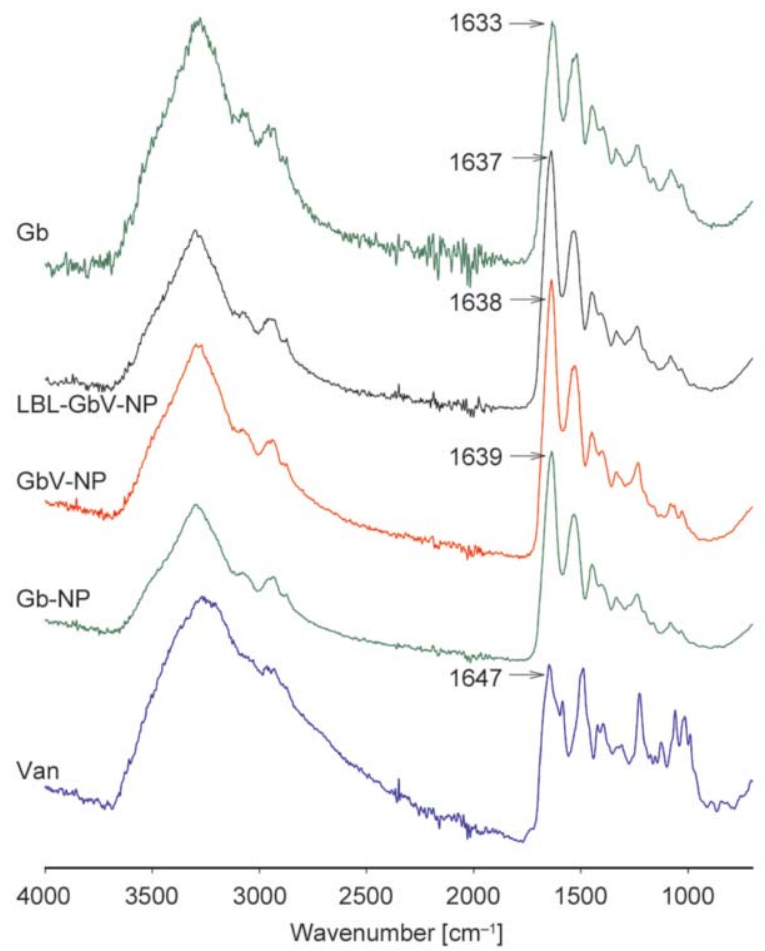

Figure 3. ATR-FTIR of vancomycin (Van) and gelatin B (Gb) powders and various types of nanoparticles ed to be able to kill $S$. aureus after its cleavage from the Ti surfaces [34]. In this study, if Van could interact with Gb-NP, it would be by non-covalent interactions and could be easily dissociated from the Ti surface since Van could be detected in the EE experiment and the release studies (section 3.3). Thus, the antibacterial activity of Van in the materials was retained.

\subsection{Drug release study}

The $\% \mathrm{EE}$ of Van in the GbV-NP as determined using Equation (1) was 70\%. Figure 4 shows the release profile of Van from the GbV-NP and LBL-GbV-NP coating on the Ti substrate. The release of Van from Ti/GbV-NP was up to $120 \mu \mathrm{g}$ but that from the Ti/ LBL-GbV-NP was about $30 \mu \mathrm{g}$ in $48 \mathrm{~h}$. This was probably due to the polyelectrolyte coating layer that was able to hinder the release of the drug. The release of Van from both the NPs demonstrated a sustained release of the drug and would be able to prolong the protection of the bone from infection.

\subsection{Antibactierial efficacy}

The antibacterial efficiency of the Ti/GbV-NP and Ti/LBL-GbV-NP against $S$. aureus which is the most common micro-organisms that causes implant-associated infections was determined [12, 34, 35]. As shown in Figure 5, clear zones were not detected for the bare Ti or the Ti with the Gb-NP coating. Hence these two materials had no antibacterial activity. Clear zones were observed from both the materials coated with Van (Ti/GbV-NP and Ti/LBL-GbV-NP). The inhibition zones that were detected after incubation for $24 \mathrm{~h}$ of Ti/GbV-NP was slightly larger than those for the Ti/LBL-GbV-NP. This was consistent with the release of Van from the coated materials and demon-

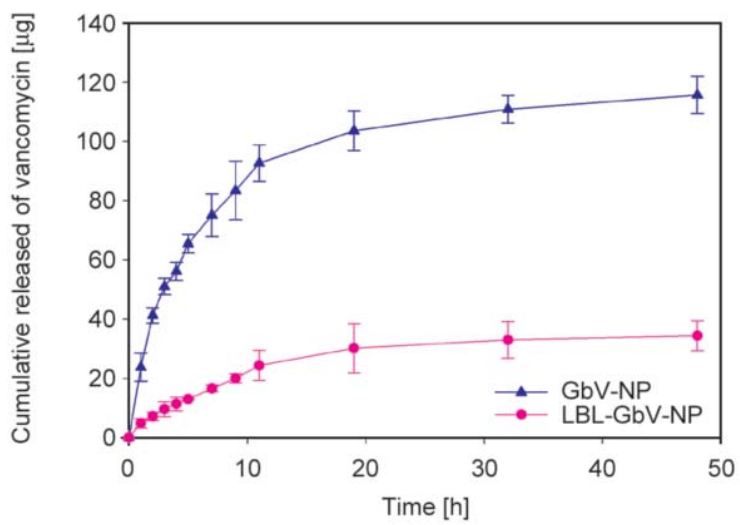

Figure 4. Release profiles of vancomycin from GbV-NP and LBL-GbV-NP coated on Ti substrate 


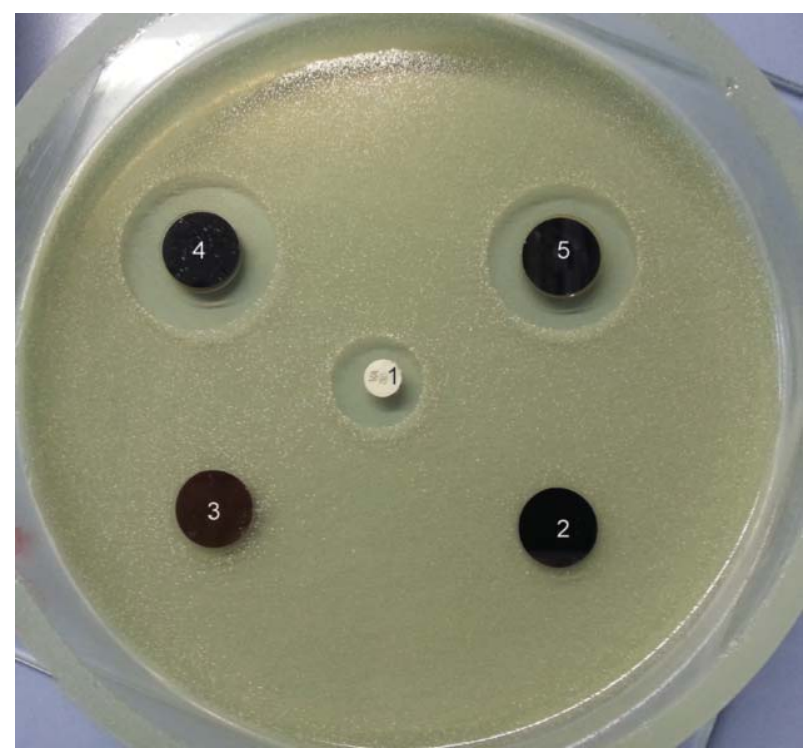

Figure 5. Inhibition zone caused by Van $(30 \mu \mathrm{g})(1)$, bare $\mathrm{Ti}$ (2) and various nanoparticle, Gb-NP (3). GbVNP (4), LBL-GbV-NP (5), coatings on Ti substrate. Based on NP preparation and \%EE, approximate $140 \mu \mathrm{g}$ of Van was loaded on the in GbV-NP and LBL-GbV-NP.

strated that both materials would effectively reduce bacterial infection.

\subsection{Cell proliferation and cell viability}

Osteoblast cells have been widely used as a model to investigate the behavior of osteoblasts on polymer surfaces [36]. In this study, cell proliferation of osteoblast like cells (CAL-72) on the bare Ti and coated materials is shown in Figure 6. Cell proliferation on the Ti/LBL-GbV-NP $\left(5.6 \cdot 10^{4}\right.$ cells $)$ was slightly

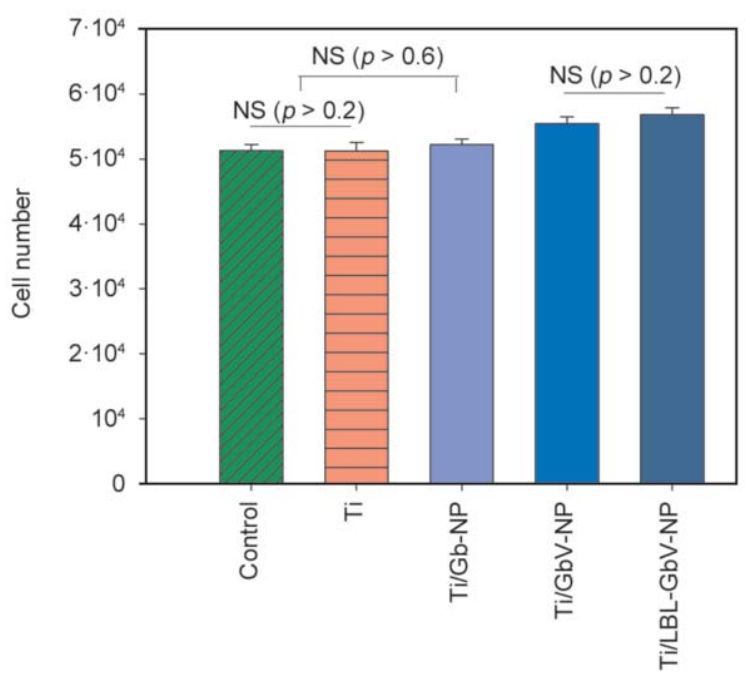

Figure 6. Proliferation of osteoblasts cultured on Ti substrate and various nanoparticle coatings on Ti substrate for 3 days (mean $\pm \mathrm{SD}, n=5$ pergroup) higher than that on the Ti/GbV-NP $\left(5.5 \cdot 10^{4}\right.$ cells $)$ but not significanly different $(p>0.2)$. However cell proliferation on both the materials was higher than that on the Ti/Gb-NP $(p<0.001)$. Cell proliferation on the bare Ti was not statistically different from that on the control $(p>0.9)$ and on the Ti/Gb-NP $(p>0.6)$.

As shown in Figure 7, the viability of the osteoblast cells on all the test samples with a culture period of 3 days showed favorable cell viability on all coated Ti: Ti/Gb-NP (105 $\pm 4.1 \%)$, Ti/GbV-NP (108 $\pm 2.9 \%)$ and Ti/LBL-GbV-NP (110 $\pm 3.9 \%)$. Cell viability on the coated materials was all very similar $(p>0.3)$. Cell viability on Ti/GbV-NP and Ti/LBL-GbV-NP was higher than that on the bare Ti $(p<0.004)$ $(100 \pm 2.9 \%)$ which was not significantly different from the control $(p>0.7)$. This cell viability result demonstrated that these coated materials were not toxic to the osteoblast like cells. In addition, the coated materials did induce more cell proliferation than the control.

The adhesion and spread of the osteoblasts over the biomaterial was the initial step of cell-material interaction and this affected the subsequent cell proliferation and/or differentiation [6]. As shown in Figure 8 , adhered cells developed more on the coated materials than on the bare Ti. This demonstrated a preferable interaction between the cells and the coated materials. Furthermore, LBL-GbV-NP coated onto the Ti showed the most attachments by the cells. Consequently, cell proliferataion on Ti/LBL-GbV-NP was higher than that on the Ti/GbV-NP and the Ti/Gb-NP.

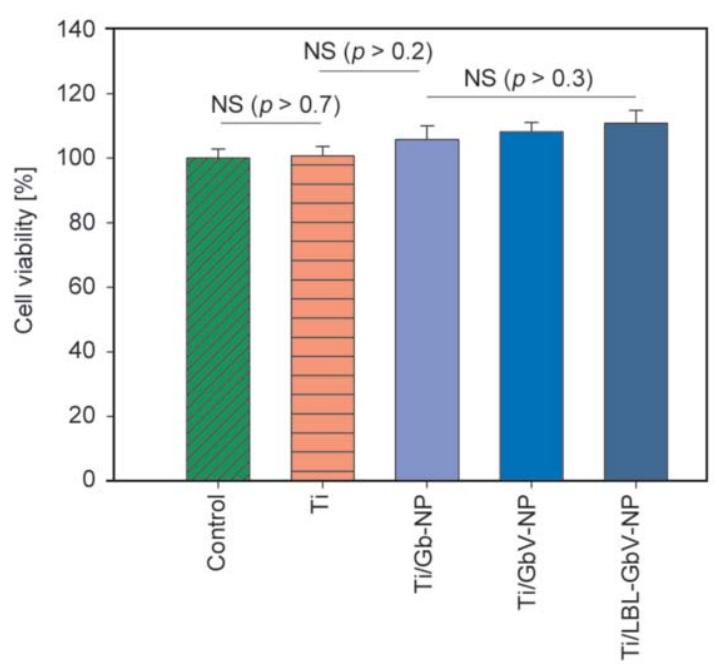

Figure 7. Cell viability of osteoblasts seeded onto Ti substrate and various nanoparticle coatings on Ti substrate for 3 days (mean $\pm \mathrm{SD}, n=5$ pergroup) 

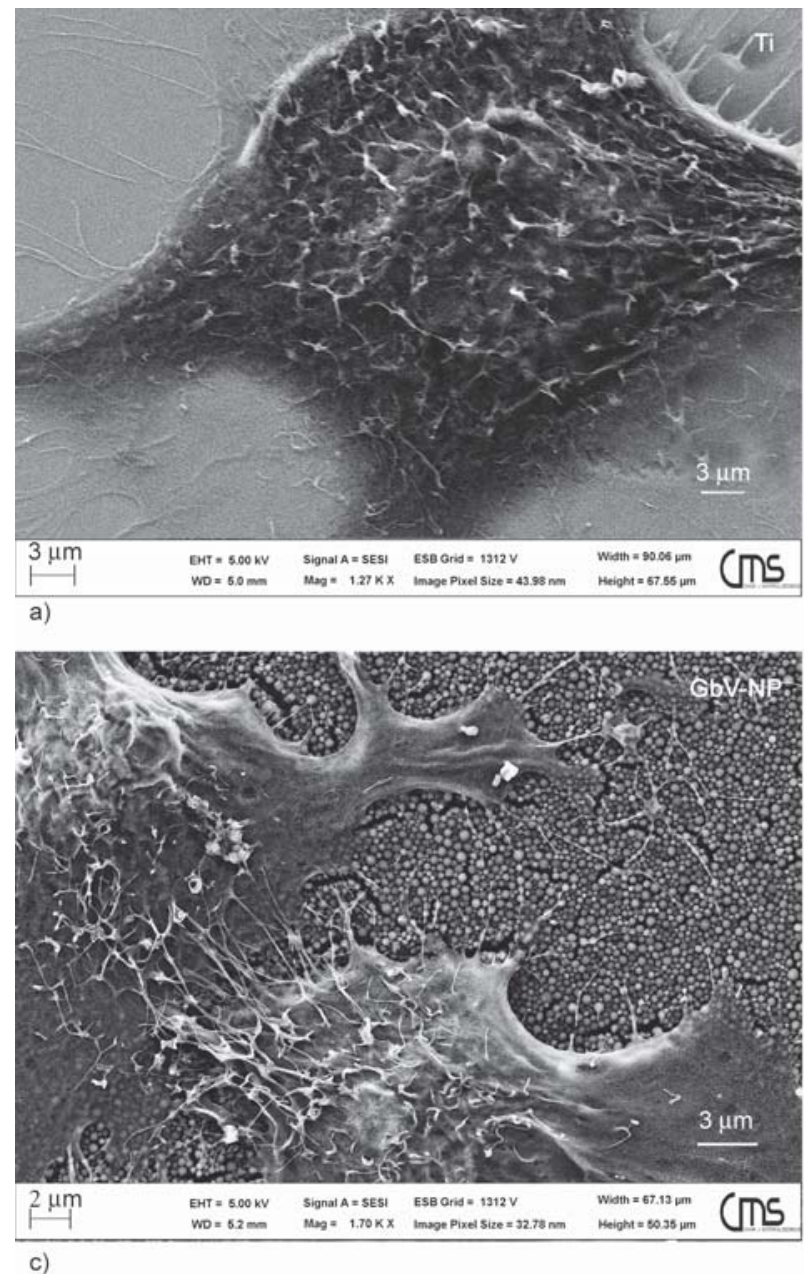

Figure 8. FIB-SEM images of osteoblast like cells on bare Ti GbV-NP (d) after cultured for 3 days

\section{Conclusions}

Modifications to the titanium surface by coating with GbV-NP and LBL-GbV-NP were prepared in order to prevent implant-associated infections. The coated materials were not cytotoxic. However, the Ti coated materials showed a significantly higher proliferation of osteoblast like cells than the bare Ti. The improvement of cell proliferation was probably due to the roughness of the NP coatings that enhanced cell attachment to the surface. The antibacterial activity of the Ti/GbV-NP was slightly higher than that of Ti/LBL-GbV-NP which agreed with the data on the release of Van from these coating materials. Moreover, the coated materials demonstrated a sustained release of Van. Thus the coated materials loaded with the drug may prevent infections for a longer period of time.
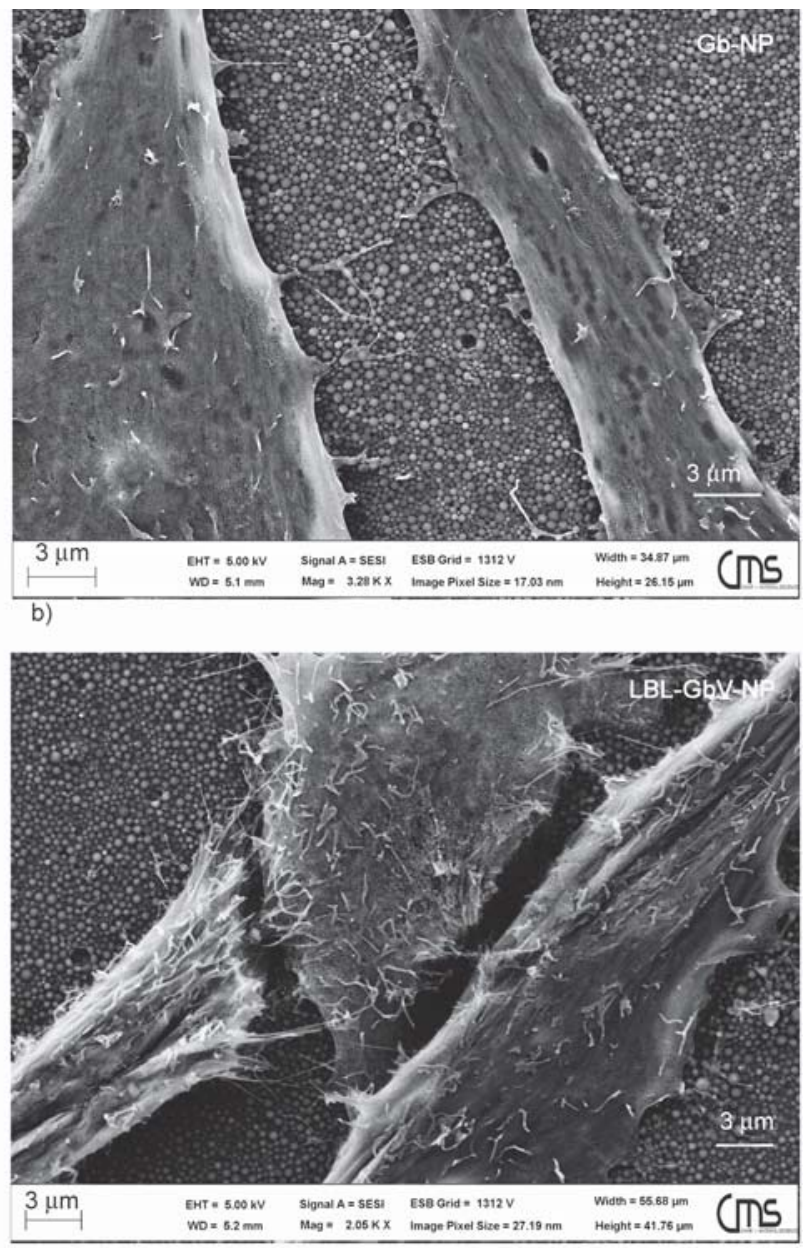

d)

\section{Acknowledgements}

This work was supported by the Thailand Research Fund through the Royal Golden Jubilee Ph.D. Program through Grant No PHD/0045/2552 and the Nanotechnology Center (NANOTEC), NSTDA, Ministry of Science and Technology, Thailand, through its program of Center of Excellence Network. Thanks also to Dr. Brian Hodgson for assistance with the English.

\section{References}

[1] Geetha M., Singh A. K., Asokamani R., Gogia A. K.: Ti based biomaterials, the ultimate choice for orthopaedic implants - A review. Progress in Materials Science, 54, 397-425 (2009).

https://doi.org/10.1016/j.pmatsci.2008.06.004

[2] Zhao L., Chu P. K., Zhang Y., Wu Z.: Antibacterial coatings on titanium implants. Journal of Biomedical Materials Research Part B: Applied Biomaterials, 91, 470480 (2009).

https://doi.org/10.1002/jbm.b.31463 
[3] Stigter M., Bezemer J., de Groot K., Layrolle P.: Incorporation of different antibiotics into carbonated hydroxyapatite coatings on titanium implants, release and antibiotic efficacy. Journal of Controlled Release, 99, 127 137 (2004).

https://doi.org/10.1016/j.jconrel.2004.06.011

[4] Swanson T. E., Cheng X., Friedrich C.: Development of chitosan-vancomycin antimicrobial coatings on titanium implants. Journal of Biomedical Materials Research Part A, 97, 167-176 (2011).

https://doi.org/10.1002/jbm.a.33043

[5] Radin S., Ducheyne P.: Controlled release of vancomycin from thin sol-gel films on titanium alloy fracture plate material. Biomaterials, 28, 1721-1729 (2007). https://doi.org/10.1016/j.biomaterials.2006.11.035

[6] Cai K., Rechtenbach A., Hao J., Bossert J., Jandt K. D.: Polysaccharide-protein surface modification of titanium via a layer-by-layer technique: Characterization and cell behaviour aspects. Biomaterials, 26, 5960-5971 (2005). https://doi.org/10.1016/j.biomaterials.2005.03.020

[7] Cai K., Hu Y., Jandt K. D.: Surface engineering of titanium thin films with silk fibroin via layer-by-layer technique and its effects on osteoblast growth behavior. Journal of Biomedical Materials Research Part A, 82, 927-935 (2007).

https://doi.org/10.1002/jbm.a.31233

[8] Cai K., Hu Y., Jandt K. D., Wang Y.: Surface modification of titanium thin film with chitosan via electrostatic self-assembly technique and its influence on osteoblast growth behavior. Journal of Materials Science: Materials in Medicine, 19, 499-506 (2008).

https://doi.org/10.1007/s10856-007-3184-5

[9] Córdoba A., Hierro-Oliva M., Pacha-Olivenza M. A., Fernandez-Calderón M. C., Perelló J., Isern B., Gonzalez-Martín M. L., Monjo M., Ramis J. M.: Direct covalent grafting of phytate to titanium surfaces through Ti-O-P bonding shows bone stimulating surface properties and decreased bacterial adhesion. ACS Applied Materials and Interfaces, 8, 11326-11335 (2016). https://doi.org/10.1021/acsami.6b02533

[10] Mattioli-Belmonte M., Cometa S., Ferretti C., Iatta R., Trapani A., Ceci E., Falconi M., De Giglio E.: Characterization and cytocompatibility of an antibiotic/chitosan/cyclodextrins nanocoating on titanium implants. Carbohydrate Polymers, 110, 173-182 (2014). https://doi.org/10.1016/j.carbpol.2014.03.097

[11] Sharma S., Bano S., Ghosh A. S., Mandal M., Kim HW., Dey T., Kundu S. C.: Silk fibroin nanoparticles support in vitro sustained antibiotic release and osteogenesis on titanium surface. Nanomedicine: Nanotechnology, Biology and Medicine, 12, 1193-1204 (2016). https://doi.org/10.1016/j.nano.2015.12.385

[12] Zhang L., Yan J. W., Yin Z. W., Tang C., Guo Y., Li D., Wei B., Xu Y., Gu Q. R., Wang L. M.: Electrospun vancomycin-loaded coating on titanium implants for the prevention of implant-associated infections. International Journal of Nanomedicine, 9, 3027-3036 (2014). https://doi.org/10.2147/ijn.s63991
[13] Das K., Bose S., Bandyopadhyay A.: $\mathrm{TiO}_{2}$ nanotubes on Ti: Influence of nanoscale morphology on bone cellmaterials interaction. Journal of Biomedical Materials Research Part A, 90, 225-237 (2009). https://doi.org/10.1002/jbm.a.32088

[14] Liu X., Chu P. K., Ding C.: Surface modification of titanium, titanium alloys, and related materials for biomedical applications. Materials Science and Engineering R: Reports, 47, 49-121 (2004). https://doi.org/10.1016/j.mser.2004.11.001

[15] Ainslie K. M., Tao S. L., Popat K. C., Daniels H., Hardev V., Grimes C. A., Desai T. A.: In vitro inflammatory response of nanostructured titania, silicon oxide, and polycaprolactone. Journal of Biomedical Materials Research Part A, 91, 647-655 (2009). https://doi.org/10.1002/jbm.a.32262

[16] Feng W., Nie W., He C., Zhou X., Chen L., Qiu K., Wang W., Yin Z.: Effect of pH-responsive alginate/chitosan multilayers coating on delivery efficiency, cellular uptake and biodistribution of mesoporous silica nanoparticles based nanocarriers. ACS Applied Materials and Interfaces, 6, 8447-8460 (2014).

https://doi.org/10.1021/am501337s

[17] Gentile P., Frongia M. E., Cardellach M., Miller C. A., Stafford G. P., Leggett G. J., Hatton P. V.: Functionalised nanoscale coatings using layer-by-layer assembly for imparting antibacterial properties to polylactide-coglycolide surfaces. Acta Biomaterialia, 21, 35-43 (2015). https://doi.org/10.1016/j.actbio.2015.04.009

[18] Du P., Zhao X., Zeng J., Guo J., Liu P.: Layer-by-layer engineering fluorescent polyelectrolyte coated mesoporous silica nanoparticles as $\mathrm{pH}$-sensitive nanocarriers for controlled release. Applied Surface Science, 345, 90-98 (2015). https://doi.org/10.1016/j.apsusc.2015.03.151

[19] del Hoyo-Gallego S., Pérez-Álvarez L., Gómez-Galván F., Lizundia E., Kuritka I., Sedlarik V., Laza J. M., VilaVilela J. L.: Construction of antibacterial poly(ethylene terephthalate) films via layer by layer assembly of chitosan and hyaluronic acid. Carbohydrate Polymers, 143, 35-43 (2016).

https://doi.org/10.1016/j.carbpol.2016.02.008

[20] Zhong X., Song Y., Yang P., Wang Y., Jiang S., Zhang X., Li C.: Titanium surface priming with phase-transited lysozyme to establish a silver nanoparticle-loaded chitosan/hyaluronic acid antibacterial multilayer via layer-by-layer self-assembly. Plos One, 11, e0146957/1-e0146957/11 (2016). https://doi.org/10.1371/journal.pone.0146957

[21] Yilmaz M. D.: Layer-by-layer hyaluronic acid/chitosan polyelectrolyte coated mesoporous silica nanoparticles as $\mathrm{pH}$-responsive nanocontainers for optical bleaching of cellulose fabrics. Carbohydrate Polymers, 146, 174180 (2016). https://doi.org/10.1016/j.carbpol.2016.03.037 
[22] Zhu X., Loh X.: Layer-by-layer assemblies for antibacterial applications. Biomaterials Science, 3, 1505-1518 (2015) https://doi.org/10.1039/c5bm00307e

[23] Yan Y., Bjönmalm M., Caruso F.: Assembly of layerby-layer particles and their interactions with biological systems. Chemistry of Materials, 26, 452-460 (2014). https://doi.org/10.1021/cm402126n

[24] Elosua C., Lopez-Torres D., Hernaez M., Matias I. R., Arregui F. J.: Comparative study of layer-by-layer deposition techniques for poly(sodium phosphate) and poly(allylamine hydrochloride). Nanoscale Research Letters, 8, 539/1-539/10 (2013). https://doi.org/10.1186/1556-276x-8-539

[25] Kozlov P. V., Burdygina G. I.: The structure and properties of solid gelatin and the principles of their modification. Polymer, 24, 651-666 (1983). https://doi.org/10.1016/0032-3861(83)90001-0

[26] Bonilla J., Fortunati E., Atarés L., Chiralt A., Kenny J. M.: Physical, structural and antimicrobial properties of poly vinyl alcohol-chitosan biodegradable films. Food Hydrocolloids, 35, 463-470 (2014). https://doi.org/10.1016/j.foodhyd.2013.07.002

[27] Ding F., Nie Z., Deng H., Xiao L., Du Y., Shi X.: Antibacterial hydrogel coating by electrophoretic co-deposition of chitosan/alkynyl chitosan. Carbohydrate Polymers, 98, 1547-1552 (2013).

https://doi.org/10.1016/j.carbpol.2013.07.042

[28] Fernández I. C. S., van der Mei H. C., Lochhead M. J., Grainger D. W., Busscher H. J.: The inhibition of the adhesion of clinically isolated bacterial strains on multicomponent cross-linked poly(ethylene glycol)-based polymer coatings. Biomaterials, 28, 4105-4112 (2007). https://doi.org/10.1016/j.biomaterials.2007.05.023

[29] Coester C. J., Langer K., von Briesen H., Kreuter J.: Gelatin nanoparticles by two step desolvation a new preparation method, surface modifications and cell uptake. Journal of Microencapsulation, 17, 187-193 (2000). https://doi.org/10.1080/026520400288427
[30] Azarmi S., Huang Y., Chen H., McQuarrie S., Abrams D., Roa W., Finlay W. H., Miller G. G., Löbenberg R.: Optimization of a two-step desolvation method for preparing gelatin nanoparticles and cell uptake studies in 143B osteosarcoma cancer cells. Journal of Pharmacy and Pharmaceutical Sciences, 9, 124-132 (2006).

[31] Le Ray A-M., Chiffoleau S., Iooss P., Grimandi G., Gouyette A., Daculsi G., Merle C.: Vancomycin encapsulation in biodegradable poly( $\varepsilon$-caprolactone) microparticles for bone implantation. Influence of the formulation process on size, drug loading, in vitro release and cytocompatibility. Biomaterials, 24, 443-449 (2003). https://doi.org/10.1016/S0142-9612(02)00357-5

[32] Bauer A. W., Kirby W. M., Sherris J. C., Turck M.: Antibiotic susceptibility testing by a standardized single disk method. American Journal of Clinical Pathology, 45, 493-496 (1966).

[33] Ofokansi K., Winter G., Fricker G., Coester C.: Matrixloaded biodegradable gelatin nanoparticles as new approach to improve drug loading and delivery. European Journal of Pharmaceutics and Biopharmaceutics, 76, 19 (2010). https://doi.org/10.1016/j.ejpb.2010.04.008

[34] Jose B., Antoci V. Jr., Zeiger A. R., Wickstrom E., Hickok N. J.: Vancomycin covalently bonded to titanium beads kills Staphylococcus aureus. Chemistry and Biology, 12, 1041-1048 (2005). https://doi.org/10.1016/j.chembiol.2005.06.013

[35] Zimmerli W., Sendi P.: Pathogenesis of implant-associated infection: the role of the host. Seminars in Immunopathology, 33, 295-306 (2011). https://doi.org/10.1007/s00281-011-0275-7

[36] Rangabhatla A. S. L., Tantishaiyakul V., Oungbho K., Boonrat O.: Fabrication of pluronic and methylcellulose for etidronate delivery and their application for osteogenesis. International Journal of Pharmaceutics, 499, 110-118 (2016). https://doi.org/10.1016/j.ijpharm.2015.12.070 